\title{
Review of: "The impact of health worker absenteeism on patient health care seeking behavior, testing and treatment: A longitudinal analysis in Uganda"
}

\author{
Louise Ackers ${ }^{1}$ \\ 1 University of Salford
}

Potential competing interests: The author(s) declared that no potential competing interests exist.

This is an extremely important paper that hits the nail on the head: health worker absenteeism lies at the heart of health systems failure in Uganda. The authors are correct to describe this as a 'chronic' problem; it is endemic. It is also very true to describe this as fundamentally a political problem; one that cannot be solved through simple accountability mechanisms.

It is particularly valuable to see quantitative research on this slippery and sensitive problem and to demonstrate, quite convincingly, the relationship between absenteeism and care-seeking behaviour. The paper also demonstrates the impact of absenteeism on Ugandan's ability to deliver Universal Health Coverage when many of the country's poorest are having to resort to private sector 'options' and significant out-of-pocket expenses. This data is very important, not only to understanding the functioning of the mixed economy of healthcare in Uganda but also the impacts on Uganda's ability to meet its commitments under the National Action Plan on Antimicrobial Resistance. Resort to private suppliers of antimalarials and antibiotics leads to over-consumption and contributes to the fast-growing resistance to commonly used antimicrobials in Uganda. This has global ramifications and confounds attempts in Ugandan public health facilities to promote effective antimicrobial stewardship.

The authors openly acknowledge some limitations to this quantitative study; it is extremely difficult and highly sensitive to conduct research in this area and discuss the findings openly and the study has produced some very important evidence. More qualitative and ethnographic work conducted over many years draws attention to some of the complexity that quantitative approaches may fail to capture. The focus on situations where no workers are present and the inability (in this data set) to distinguish cadres, inevitably identifies lower-level health facilities as the key problem. This is not the case. Absenteeism at Regional Referral Hospitals and higher-level health centres is rife particularly amongst senior doctors. Medical absenteeism can reach levels of $85 \%$ or more and the presence of doctors is often entirely unpredictable. This contributes significantly to maternal mortality in Uganda (https://blogs.bmj.com/bmj/2018/06/28/maternal-mortality-in-low-resource-settings-are-doctors-part-of-thesolution-or-the-problem/). The absence of senior staff - who are also often the in-charges at facilities generates a culture of absenteeism. Absenteeism should also be linked directly to late-coming and earlyleaving with many health facilities effectively only open in the morning and unable to deliver 
comprehensive maternity care when staff refuse to cover night duties and weekends.

Whilst evidencing alarming trends the quantitative data cannot being to capture causal factors - that may help to define solutions. The first cause is simple; because they can. There is no effective system in the Ugandan public sector to discipline health workers and very senior (and much more highly remunerated) cadres are more absent than lower cadres. It is not simply a matter of pay but amongst lower cadres' salaries in Uganda are below subsistence level. Most health workers have to carry 2 or more positions to survive. This absolute poverty at one end of the spectrum bleeds into abject greed at the other with highly paid cadres able to establish lucrative private businesses and even utilise public facilities to generate income. The system turns a blind eye to this behaviour.

Pay is only one factor; many health facilities in Uganda are so badly equipped and crippled by supply chain problems that health workers who do come to work are unable to deliver anything approaching safe and high-quality care. They are also often at personal risk due to the lack of genuine attention to infectionprevention-control and consumable supplies. This situation has a devastating impact on health worker motivation.

Foreign intervention often further contributes to absenteeism; an obsession with 'training' takes (often more senior) staff away from clinical duties attracted by damaging per diems. Volunteers are often permitted to substitute for local staff in ways that encourage absenteeism and undermine local human resource management (Ackers and Ackers-Johnson, 2016).

I hope the paper stimulates a greater interest, in global health agenda, on the issue of human resource management and a more open and honest debate so that the Ugandan health system can move forward. 